\title{
Time to recovery and determinants of severe acute malnutrition among 6-59 months children treated at outpatient therapeutic programme in North Gondar zone, Northwest Ethiopia: a prospective follow up study
}

Worku Nigussu Mamo ${ }^{1}$, Terefe Derso ${ }^{2}$, Kassahun Alemu Gelaye ${ }^{3}$ and Temesgen Yihunie Akalu ${ }^{3^{*}}$

\begin{abstract}
Background: Despite the available interventions to tackle nutritional problems, there is scarce information on time to recovery and its determinants among children with SAM in Ethiopia.

Objective: This study was aimed at finding the time to recovery and determinants among 6-59 months children with severe acute malnutrition treated at an outpatient therapeutic programme in North Gondar zone, northwest Ethiopia.
\end{abstract}

Methods: Facility based prospective follow up study was conducted from March 24 to May 24, 2017. A total of 408 children with the age of 6-59 months were included in the study. Structured interviewer administered questionnaire was used. Anthropometric measurements were conducted every week. The median time of recovery, Kaplan Meier (KM) curve, and log rank test were computed. Both bi-variable and multivariable Cox regression model was fitted. To establish an association between time to recovery and its determinants $95 \%$ confidence interval $(\mathrm{Cl})$ and $p$-value $<0.05$ were used. Proportional hazard assumption was checked graphically and using Schoenfeld residual test.

Results: Out of 389 children, 254 (65.3\%) recovered. The median time to recovery was $38.5 \pm$ IQR of 14 days. Children with diarrhoea $\mathrm{AHR}=0.81$ with $95 \% \mathrm{Cl}(0.73,0.99)$, children taken amoxicillin $\mathrm{AHR}=2.304$ with $95 \% \mathrm{Cl}$ (1.68-3.161), and had vomiting at admission $A H R=0.430$ with $95 \% \mathrm{Cl}(0.205,0.904)$ were significant predictors of time to recovery.

Conclusions and recommendations: The overall time to recovery has not met the minimum sphere international standard which was lower than $75 \%$. It is advisable to give emphasis to patients with diarrhoea and vomiting.

Keywords: SAM, Time to recovery, North Gondar

\footnotetext{
* Correspondence: temesgenyihunie@gmail.com

${ }^{3}$ Department of Epidemiology and Biostatistics, Institute of Public Health,

College of Medicine and Health Sciences, University of Gondar, Gondar,

Ethiopia

Full list of author information is available at the end of the article
}

(c) The Author(s). 2019 Open Access This article is distributed under the terms of the Creative Commons Attribution 4.0 International License (http://creativecommons.org/licenses/by/4.0/), which permits unrestricted use, distribution, and reproduction in any medium, provided you give appropriate credit to the original author(s) and the source, provide a link to the Creative Commons license, and indicate if changes were made. The Creative Commons Public Domain Dedication waiver (http://creativecommons.org/publicdomain/zero/1.0/) applies to the data made available in this article, unless otherwise stated. 


\section{Introduction}

Severe acute malnutrition (SAM) is defined as, a weightfor-height measurement of $\leq 70 \%$, weight for height $\mathrm{Z}$ score $<3$ Standard deviation (SD), presence of bilateral pitting edema, and/or a mid-upper-arm circumference of $<110 \mathrm{~mm}$ (MUAC) in children age 6-59 months [1].

According to global report acute malnutrition is a major cause of death among under-five children. Globally, in 2011 an estimated of 2 million children suffered from SAM. Out of them more than $80 \%$ were treated in four sub-Saharan Africa countries (Ethiopia (27.9\%), Niger (27.6\%), Somalia (15.4\%), and Democratic Republic of Congo (14.5\%) [2]. According to current estimates, approximately 17 million children under the age of 5 years were suffer from SAM and nearly two-thirds were from Southeast Asia and sub-Saharan Africa [3]. According to 2016 EDHS report, children who were wasted $<-2$ SD were 9.9\%. Similarly, the prevalence of severe wasting in Ethiopia and Amhara region was 2.9 and $2.2 \%$, respectively [4].

SAM has a high case fatality rate despite use of available management protocols [5]. According to a systematic review and meta-analysis from low and middle income countries on the treatment of severe and moderate acute malnutrition the case fatality rate for inpatient treatment of SAM using World Health Organization (WHO) protocol ranged from 3.4 to $35 \%$ [5].

According to previous studies there are several factors contributing for time to recovery among SAM patients. Low weight gain, diarrhoea $[6,7]$, education and training of health worker [8], antibiotics [9], rickets [10, 11], pneumonia [11], malnutrition status [1], stabilization centers [1], age [12], put on anti-biotic (Amoxicillin) [13], sharing of ready to use therapeutic food (RUTF) [14], being vaccinated [15], children receiving water treatment [16] and poor appetite [17] were important predictors of time to recovery among SAM patients.

Despite the use of available guidelines for management of SAM, co-morbidities and poor treatment outcomes are still observed in therapeutic feeding centers [11]. However, studies on time to recovery were very limited in Ethiopia including the study area. Therefore, this study aimed at determining the time to recovery and its predictors among children with SAM in North Gondar zone, Ethiopia.

\section{Methods and materials}

\section{Study design and setting}

Health facility based prospective follow up study was conducted from March 24 to May 24, 2017. The study was conducted in five districts of North Gondar zone including: Dabat, Wogera, Gondar zuriya, West Armachiwo, and Tach Armachiwo districts. Seventeen health centers and
31 health posts provided OTP service in the selected health facilities.

\section{Population, sample size, and sampling procedure}

All SAM children between 6 and 59 months and admitted in the selected health facilities were included in the study. Sample size was determined using study conducted from Enderta district, Tigray, Northern Ethiopia, 2012 [18]. So, we calculate the sample size by medcalc@version 18.11.3 survival analysis (logrank test) at http://www.medcal.org [17]. Adding 5\% non response rate and design effect of 1.5 the final sample size was 408 (Table 1 ).

A multi-stage random sampling technique was applied. Among 22 districts 5 were selected by using simple random sampling technique. In these 5 districts, 48 health facilities had been provided OTP services. Only health centers and health posts running OTP service in the selected districts were included in the study.

\section{Data collection tool and procedure}

A structured questionnaire was developed in English and it translated to the local Amharic language and back translated to English language to check its consistency. Structured interviewer administered questionnaire was used to collect information from each study participant. Mothers or caregivers of the selected children were interviewed. Anthropometric measurements and physical examination were used to collect data from study participants. Body weight was measured using a $25 \mathrm{~kg}$ hanging spring scale to the nearest $0.1 \mathrm{~kg}$ for children below the age of 3 years. For children less than $85 \mathrm{~cm}$, the measuring board was placed on the ground and read to the nearest $0.1 \mathrm{~cm}$ in the recumbent position. On the other hand, for children more than $85 \mathrm{~cm}$, the measuring board was fixed where the ground is level by standing position and read to the nearest $0.1 \mathrm{~cm}$. Similarly, MUAC was measured on the left upper arm of a child and its value was recorded to the nearest $1 \mathrm{~mm}$. Measurements like medical complications and presence of bilateral pitting edema were recorded on admission and at follow-up on a standard individual treatment card. Appetite test was conducted every week in a quite environment on each visit for child enrolled in the program. A child was said to pass the appetite test when she or he was able to consume the amount of RUTF recommended for her or his body weight. Children who failed the appetite test in any visit were referred to inpatient care. At admission, the data collectors assessed the degree of pitting edema, hydration, dysentery, diarrhoea, anemia, and other signs of infections. Each participant on OTP was visiting to their closest site weekly to receive food and a medical assessment. During every visit, the child was examined and given a weekly supply 
Table 1 Sample size determination using logrank test by medcalc@version 18.11.3

\begin{tabular}{|c|c|c|c|c|c|c|c|c|}
\hline Variables & & Cured & Censored & $\%$ of outcome & AHR & Log rank & $p$-value & Total event needed \\
\hline \multirow[t]{2}{*}{ Distance of $\mathrm{HI}$ from residence } & $<2 h$ & 196 & 46 & 72.9 & 1.48 & 19.3 & $<0.001$ & 258 \\
\hline & $>2 h$ & 55 & 31 & 65.5 & 1 & & & \\
\hline \multirow[t]{2}{*}{ Storage of drinking water } & Bucket & 193 & 35 & 84.6 & 1.51 & 21.8 & 0.008 & 101 \\
\hline & Pot & 62 & 42 & 59.6 & 1 & & & \\
\hline \multirow[t]{2}{*}{ Sex of the child } & Male & 124 & 34 & 78.4 & 1.30 & 12.6 & 0.043 & 25 \\
\hline & Female & 13 & 43 & 23.2 & 1 & & & \\
\hline \multirow[t]{2}{*}{ Age of the child on OTP } & $<18$ months & 176 & 39 & 81.8 & 1.20 & 18.3 & 0.259 & 72 \\
\hline & $>18$ months & 79 & 81 & 49.5 & 1 & & & \\
\hline \multirow[t]{2}{*}{$\mathrm{Wt} / \mathrm{Ht}$ at admission } & $<60 \%$ & 37 & 171 & 17.8 & 1 & 27.1 & 0.001 & 24 \\
\hline & $60 \%$ & 218 & 60 & 78.4 & 1.87 & & & \\
\hline \multirow[t]{2}{*}{ Way food preparing for $<5$ children } & Separately for them & 178 & 28 & 86.4 & 1.24 & 5.9 & 0.117 & 95 \\
\hline & Together with adult & 77 & 49 & 61.1 & 1 & & & \\
\hline
\end{tabular}

RUTF. At each follow-up visit weight of the child, existence/ extent of pitting edema, presence of disease, drugs prescribed and outcome (death, discharge cured, default, or transfer) had been recorded on patient treatment cards and in the programme register.

Two days of training on the objectives of the study and how to interview, measure, and fill the questionnaire was given to the data collectors and supervisors before the actual data collection. Forty eight BSC nurses who took training on OTP and currently working in OTP sites were recruited to collect the required information. The principal investigator and three health extension workers supervised the data collection process. Besides, the data collection tool was pretested with $20(5 \%)$ of samples in Dembia district. Lastly, cleaning was done on daily basis and timely feedback was communicated to the data collectors.

\section{Measurements and study variables}

Time to recovery, the outcome variable of this study was defined as a time from admission date to discharge date while the child is cured. Time to recovery was measured by subtracting the date of admission from the discharge date. Therefore, children who were reached the discharge criteria means $(\mathrm{W} / \mathrm{L}>=85 \%$ or $\mathrm{W} / \mathrm{H}>=85 \%$ on more than one occasion for children with Marasmus, and if edema was disappeared regardless of their body weight status within 14 days for kwashiorkor cases. Whereas, children who were admitted and treated at OTP and discharged out other than cured like transfer out, unknown, defaulter, death, or non-response and still they were in a program were censored. Sociodemographic variables: age of the mother/caregiver, sex of the child, marital status, residence, occupational status of mother's or care giver, relation to child, ethnicity, income, educational status of mother's or care giver; Maternal and child health related factors: child breastfeed status, deworming, bed net utilization, type of admission, routine medication during admission and follow up, medical problems during follow-up; Hygiene and sanitation factors: Source of drinking water, Latrine, housing condition were assessed. Average length of stay was defined as sum of number of days for each recovered patients per total number of patients in a program. Weight gain $(\mathrm{g} / \mathrm{kg} /$ day): was defined as an average weight (in gram) increase for every $\mathrm{Kg}$ of body weight of the child per day.

\section{Data processing and analysis}

Data were entered into Epi-Data version 3.1 and exported to STATA 14 for analysis. Descriptive measures like percentages and median were used to describe categorical variables and continuous variables, respectively. Other descriptive measure like life-table was computed. Proportional Hazard Assumption (PHA) was checked both graphically and using Schoenfeld residual tests. Cox regression model was computed for both bi-variable and multivariate analysis and final results were taken as significance at 5\% level of significance. Adjusted hazard ratio (AHR) with its respective 95\% confidence interval (CI) was reported to show the strength of association.

\section{Socio-demographic characteristics of mothers/caregivers}

A total of 408 patients were included in the study with a response rate of $95.34 \%$. Mean age of the mother/caregiver was 31.25 with $\mathrm{SD}$ of \pm 9.8 . Among admitted patients at OTP, $65.04 \%$ were from urban residence. Majority, 238 (61.18\%) were housewives. About threefourth of respondents were illiterate (Table 2).

\section{Maternal and child related factors}

About 311 (80.21\%) were still breastfeeding their child at the time of data collection. About one-third (33.16\%) of the children were fully immunized and 140 (36.00\%) 
Table 2 Socio-demographic and economic characteristics of the mothers/guardians of children who were admitted at OTP in North Gondar, northwest Ethiopia, $2017(n=389)$

\begin{tabular}{lll}
\hline Variables & Frequency & Percentage(\%) \\
\hline Residence & 253 & 65.04 \\
Urban & 136 & 34.96 \\
Rural & & \\
Marital status & 301 & 77.38 \\
Married & 56 & 14.39 \\
Single & 19 & 4.88 \\
Divorced & 13 & 3.35 \\
Widowed & & \\
Mother's/care givers occupation & 238 & 61.18 \\
House wife & 98 & 25.19 \\
Government employee & 18 & 4.63 \\
Farmer & 26 & 6.68 \\
Merchant & 9 & 2.32 \\
Others & & \\
mother's/care giver education & & 74.04 \\
Illiterate & 288 & 25.96 \\
Literate & 101 & 72.75 \\
Relation to child & & 10.79 \\
Mother & 283 & \\
Sibling & 42 & \\
Caregiver & & \\
Grand mother & & \\
Other & & \\
\hline & & \\
& &
\end{tabular}

were not yet vaccinated. Regarding with merits of breastfeeding nearly one-fourth (25.96\%), 164 (42.16\%), 100 (25.71\%) respond as breastfeeding is important for child growth, child health, and child food, respectively. About $70 \%$ children initiated complementary feeding at 6 months (Table 3).

\section{Health service utilization}

Almost a quarter of children had medical related problems 2 weeks prior to the survey. Out of the total admissions, a total of 355 (91.26\%) were newly admitted patients, $6.17 \%$ transferred in and 10 (2.57\%) were readmitted. Among the total admitted cases, 9.3 had edema, no danger sign was looked, and a few had symptoms of diarrhea (11.82\%), vomiting (3.09\%), and cough (2.57\%) at admission. At admission, 67.9\%, 24.7\%, and $13.6 \%$ were took amoxicillin, measles, and vitamin A capsule, respectively (Table 4 ).

\section{Clinical characteristics of children}

Majority, 90.75\% patients had sign of Marasmus. During follow-up children with SAM complicated with respiratory
Table 3 Maternal health practice during treatment of severe acute malnutrition among children at OTP in North Gondar, northwest Ethiopia, 2017 ( $n=389)$

\begin{tabular}{lcl}
\hline Variables & Frequency & Percentage (\%) \\
\hline Child breastfeeding status & 77 & 19.79 \\
No & 311 & 80.21 \\
Yes & & \\
Immunization & 129 & 33.16 \\
Fully immunized & 120 & 30.84 \\
Partially immunized & 140 & 36.00 \\
Not yet vaccinated & 101 & \\
Merits of breastfeeding for mother and child & 25.96 \\
For child growth & 164 & 42.16 \\
For child health & 100 & 25.71 \\
For child food & 19 & 4.88 \\
For comfort & 5 & 1.29 \\
Other** & 17 & \\
Starting period of complementary foods & 4.37 \\
0-3 months & 44 & 11.32 \\
$4-5$ months & 268 & 68.89 \\
At 6 months & 30 & 7.71 \\
> = 7 months & 30 & 7.71 \\
I don't know & &
\end{tabular}

I don't know* not checked about his/her breastfeeding status during interviewed.

Other** the merits for mental health, for skill, knowledge

distress $6.68 \%$, acute diarrhea $17.48 \%$, and hyperthermia $2.84 \%$ and anemia accounts $1.03 \%$. Children with severe acute malnutrition checked for any problems and only $3.86 \%$ of children reassured during follow-up (Table 5).

\section{Treatment outcomes of children with severe acute malnutrition}

From the total study subjects, 254 (65.3\%) successfully recovered from SAM within the first 8 weeks of treatment and 135 (34.7\%) were censored. Among SAM children 4 (1.03\%) didn't know their status. A total of 39 (10.02\%) patients were transferred out to nearby health facility, and $2(0.51 \%)$ cases were referred to inpatient due to complications during the follow-up visits. About $17(4.37 \%)$ cases were defaulters. The average rate of weight gain among recovered children was $5.814 \mathrm{~g} / \mathrm{kg} /$ day $( \pm 2.2833)$.

\section{Survival analysis}

The participants were followed for a total of 13,104 person days (35.9 person years) observation. The median time to recovery was ( $38.5 \pm$ IQR 14 days). The probability of survival at 4 th, 5th, 6th, 7th, 8th,and 9th weeks were $85.6,55.2,35.3,13.1,5$ and $2.1 \%$, respectively. 
Table 4 Health service utilization of children who were admitted at OTP in North Gondar, northwest Ethiopia, 2017 ( $n=389)$

\begin{tabular}{|c|c|c|}
\hline Variables & Frequency & Percentage(\%) \\
\hline \multicolumn{3}{|l|}{ Types of admission } \\
\hline New & 355 & 91.26 \\
\hline Transferred in & 24 & 6.17 \\
\hline Readmitted & 10 & 2.57 \\
\hline \multicolumn{3}{|l|}{ Routine medication } \\
\hline Amoxicillin & & 67.86 \\
\hline Folic acid & & 1.03 \\
\hline Measles & & 24.68 \\
\hline Albendazol/mebendazol & 264 & 23.39 \\
\hline Vitamin A supplementary & 264 & 13.62 \\
\hline \multirow[t]{5}{*}{ Anti-Malarial drugs } & 4 & 2.06 \\
\hline & 96 & \\
\hline & 91 & \\
\hline & 53 & \\
\hline & 8 & \\
\hline \multicolumn{3}{|l|}{ Diarrhea at admission } \\
\hline Yes & 46 & 11.82 \\
\hline No & 343 & 88.18 \\
\hline \multicolumn{3}{|l|}{ Vomiting at admission } \\
\hline Yes & 12 & 3.09 \\
\hline No & 377 & 96.91 \\
\hline \multicolumn{3}{|l|}{ Cough at admission } \\
\hline Yes & 10 & 2.57 \\
\hline No & 379 & 97.43 \\
\hline \multicolumn{3}{|l|}{ Diarrhea during follow up } \\
\hline Yes & 8 & 2.06 \\
\hline No & 381 & 97.94 \\
\hline \multicolumn{3}{|l|}{ Fever during follow up } \\
\hline Yes & 9 & 2.31 \\
\hline No & 380 & 97.69 \\
\hline \multicolumn{3}{|l|}{ Vomiting during follow up } \\
\hline Yes & 9 & 2.3 \\
\hline No & 380 & 97.7 \\
\hline \multicolumn{3}{|l|}{ Cough during follow up } \\
\hline Yes & 25 & 6.43 \\
\hline No & 364 & 93.57 \\
\hline
\end{tabular}

The proportional hazard assumption was checked by the Schoenfeld residual global test, and $p$-value ( $p=$ $0.1464)$. So, proportional hazard assumption was met. Goodness of fit for the fitted model was also performed using the Cox Snell residual test and showed that the model was adequate.

Time to recovery for patients who had vomiting on admission a median recovery time was 56 days, whereas
Table 5 Clinical characteristics of children who were admitted at OTP in North Gondar, northwest Ethiopia, 2017( $n=389)$

\begin{tabular}{lcl}
\hline Variables & Frequency & Percentages \\
\hline Sign of malnutrition & 353 & \\
Marasmus & 36 & 90.75 \\
Kwashiorkor & & 9.25 \\
Complications during follow-up & & \\
Respiratory distress & 26 & 6.68 \\
Acute diarrhea & 68 & 17.48 \\
Hyperthermia & 11 & 2.84 \\
Anemia & 4 & 1.03 \\
Admission MUAC & & \\
$<110$ mm & 235 & 63.34 \\
$\geq 110$ mm & 154 & 36.66 \\
Appetite test done at any visit consistently & \\
Yes & 379 & 97.43 \\
No & 10 & 2.57 \\
For any problems during follow up & \\
Action taken & 15 & 3.86 \\
Not Action taken & 299 & 76.86 \\
Not assessed at all & 75 & 19.28
\end{tabular}

who did not have vomiting on admission had a median recovery times of 42 days and the difference was significant ( $p$-value $=0.038)$. Similarly, $60 \%$ of patients with vomiting still not recovered at 49 days as compared to $12.2 \%$ of patients without vomiting (Fig. 1.)

\section{Predictors of time to recovery among SAM patients}

In the bi-variate analysis place of residence, sex, diarrhoea on admission, vitamin $\mathrm{A}$, amoxicillin intake at admission, providing folic acid at admission, vomiting at admission and provision of deworming during follow-up were significantly associated with time to recovery. However, in the multi-variable Cox regression model diarrhea at admission, vomiting at admission, providing folic acid at admission, and provision of Amoxicillin at admission remained statistically significant predictors of time to recovery.

The hazard of patients with vomiting at admission prolonged time to recovery by $57 \%$ compared to those patients without vomiting AHR $=0.430$ at CI $95 \%(0.205$, 0.904). Similarly, the rate of time to recovery among who had diarrhea on admission delayed recovery time by $19 \%$ compared to patients who had no diarrhea (AHR $=0.811 ; 95 \%$ CI:0.735-0.993).

The rate of time to recovery among patients taken amoxicillin at admission was 2.304 times faster recover from SAM as compared to they did not took it $(\mathrm{AHR}=$ 2.304; 95\%CI:1.680-3.161) (Table 6). 


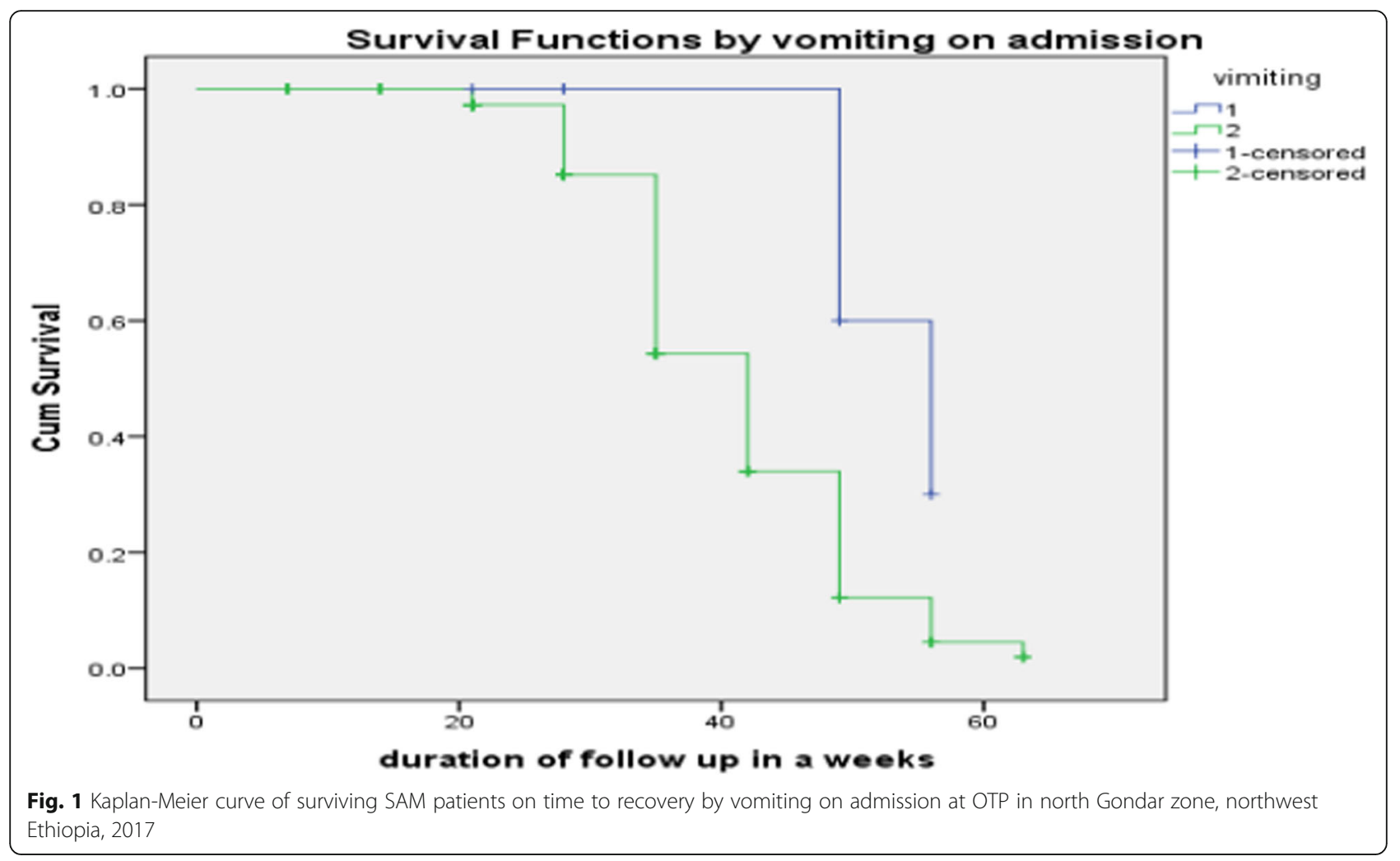

\section{Discussion}

This study assessed time to recovery of severely malnourished children aged 6-59 months managed on outpatient basis for a maximum duration of 8 weeks. The recovery rate was about $65.3 \%$. This finding was lower than the sphere standard which states recovery rate should be greater than $75 \%$.

The median length of stay was ( $38.5 \pm$ IQR 14 days) for recovered/cured children and this study was found to be in line with a multicentre, randomized intervention study done in Mali which showed that median recovery time of the entire cohort was 42 days. Another prospective cohort study conducted in southern people, nation and nationalities region of Ethiopia showed that the median recovery time was 49 days $[18,19]$. However, the finding of this study found to be lower duration of stay in the outpatient therapeutic program than 92 days length of time to recovery a study done in Afar, Ethiopia [20]. The difference showed because of giving a monthly supply of therapeutic food in an area when children enroll into OTP on admission and appetite test was not checked and inappropriate quantity or inadequate to their weight of RUTF provided to them. This leads to prolonged duration of time to recover from SAM.

This finding is supported by another study done from India with median time of 51 days with IQR of 5.6 days [21]. However, the finding of this study was found to be lower than a retrospective study conducted from Zambia with 24 weeks [22] and a prospective cohort study in rural Ethiopia with median time of 9 weeks and IQR of 4-15 weeks [23]. The difference could be due to no access to supplementary feeding, intervention protocol difference in a setting, differences discharge criteria.

Children who were taken amoxicillin as routine medicines on admission had better time to recovery as compared to those who were not taken the medication at admission. This is supported by study from Wolayita zone, Ethiopia [24]. However, findings from Kambata, south Ethiopia [25] showed that taking Amoxicillin had no difference in time to recovery among SAM patients.

Children with vomiting at admission complicate SAM and prolonged time to recovery. This finding is supported by the study conducted in Tigray, Ethiopia $[8,26]$.

Diarrhea at admission is a negative predictor of timeto-recovery from SAM. The time to recovery from OTP among children who had diarrhea during admission was delayed by $19 \%$. This difference could be that diarrhea is linked to delay time to recovery and poor health outcomes of children with SAM. Similarly, the findings from rural Bangladesh suggest that diarrhea complicate and result in unfavorable nutritional consequences [27].

This study has its own strength and limitations. Since the study design was prospective follow up study we used a primary data and temporality issue was clearly ascertained. Therefore, cause and effect relationship is possible to establish. The response related to RUTF 
Table 6 Multi-variable Cox regression model among children with SAM at OTP in North Gondar zone, northwest Ethiopia, 2017( $n=389)$

\begin{tabular}{|c|c|c|c|c|}
\hline \multirow[t]{2}{*}{ Variables } & \multicolumn{2}{|c|}{ Outcome } & \multirow[t]{2}{*}{ CHR with $95 \% \mathrm{Cl}$} & \multirow[t]{2}{*}{ AHR with $95 \% \mathrm{Cl}$} \\
\hline & Event & Censored & & \\
\hline \multicolumn{5}{|l|}{ Residence } \\
\hline Urban & 175 & 78 & $1.247(1.12,1.634)$ & $0.837(0.559,1.25)$ \\
\hline Rural & 79 & 56 & 1 & 1 \\
\hline \multicolumn{5}{|l|}{ Sex } \\
\hline Male & 122 & 63 & $0.82(0.672,1.089)$ & $0.845(0.643,1.11)$ \\
\hline Female & 132 & 72 & 1 & 1 \\
\hline \multicolumn{5}{|c|}{ Diarrhea on admission } \\
\hline Yes & 51 & 17 & $0.80(0.682,0.997)$ & $0.81(0.735,0.99)^{*}$ \\
\hline No & 203 & 118 & 1 & 1 \\
\hline \multicolumn{5}{|c|}{ Vomiting at admission } \\
\hline Yes & 10 & 2 & $0.473(0.25,0.894)$ & $0.43(0.205,0.904)^{*}$ \\
\hline No & 244 & 133 & 1 & 1 \\
\hline \multicolumn{5}{|c|}{ Amoxicillin intake at admission } \\
\hline Yes & 155 & 109 & $1.96(1.504,2.544)$ & $2.304(1.680,3.161)^{*}$ \\
\hline No & 99 & 26 & 1 & 1 \\
\hline \multicolumn{5}{|c|}{ Folic acid at admission } \\
\hline Yes & 56 & 40 & 1 & 1 \\
\hline No & 198 & 95 & $0.604(0.456,0.82)$ & $0.60(0.399,0.915)^{*}$ \\
\hline \multicolumn{5}{|c|}{ Vitamin $\mathrm{A}$ at admission } \\
\hline Yes & 35 & 19 & $1.348(1.114,1.54)$ & $1.33(0.841,2.107)$ \\
\hline No & 219 & 117 & 1 & 1 \\
\hline \multicolumn{5}{|c|}{ Deworming during follow up } \\
\hline Yes & 7 & 1 & $1.426(1.237,1.73)$ & $1.09(0.386,3.08)$ \\
\hline No & 247 & 134 & 1 & 1 \\
\hline
\end{tabular}

* show statisticall significance association

shared by other siblings or not might be affected by social desirability bias. Additionally, household and environmental factors of the care taker were not addressed. Other co-morbid conditions like Tuberculosis (TB), HIV, chronic conditions like cardiac and renal abnormality of the children were not assessed.

\section{Conclusions}

The time to recovery has not met the minimum sphere international standard which was $>75 \%$ and a maximum of 8 weeks. Factors that prolong time-to-recovery include diarrhea and vomiting on admission. On the hand, routine medicines provision like amoxicillin on admission result in faster time to recovery from SAM.

\section{Acknowledgements}

We would to thank all selected health facilities for their cooperation and permission to conduct the study. We are also grateful to all respondents, data collectors, and supervisors for providing valuable time and necessary information for this research work without them the study was not materialized.

\section{Author's contribution}

WN: conceptualization, data curation, formal analysis, funding acquisition, resource, software, validation, writing original draft and writing review and editing. TD: conceptualization, formal analysis, methodology, supervision, validation, writing original draft and writing review and editing. TYA: conceptualization, data curation, formal analysis, methodology, software, supervision validation, visualization, writing original draft and writing review and editing. KAG: conceptualization, data curation, supervision, validation, visualization, writing original draft and writing review and editing. All authors read and approved the final manuscript.

\section{Funding}

No funding was obtained for this study.

Availability of data and materials

Data will be available upon request from the corresponding author.

\section{Ethics approval and consent to participate}

The study approval was obtained from the Institutional Review Board (IRB) of the University of Gondar, College of Medicine and Health Sciences, Institute of Public Health. Official letter of co-operation was also written to the selected districts and permission was obtained from each respective health facilities. Oral consent was taken from every mother or care giver before the interview by explaining the objective of the research. The study did not given any incentive to the participants as compensation for involving in the study. Any respondent's identifiers were not recorded to keep the confidentiality of the information. The questionnaire was kept securely in locked cabinets and the data base was password protected.

\section{Consent for publication}

Not applicable.

\section{Competing interests}

The authors declare that they have no conflict of interest.

\section{Author details}

${ }^{1}$ Abrhajira Primary Hospital, West Gondar Zone, Ethiopia. ${ }^{2}$ Department of Human Nutrition, Institute of Public Health, College of Medicine and Health Sciences, University of Gondar, Gondar, Ethiopia. ${ }^{3}$ Department of Epidemiology and Biostatistics, Institute of Public Health, College of Medicine and Health Sciences, University of Gondar, Gondar, Ethiopia.

Received: 20 June 2019 Accepted: 14 October 2019

Published online: 04 November 2019

\section{References}

1. Gebremichael DY. Predictors of nutritional recovery time and survival status among children with severe acute malnutrition who have been managed in therapeutic feeding centers, Southern Ethiopia: retrospective cohort study. BMC Public Health. 2015;15:1267.

2. UNICEF. Improving child nutrition the achievable imperative for global progress 2013.

3. Yaya S, Bishwajit G, Ekholuenetale M, Shah V, Kadio B, Udenigwe O. Timing and adequate attendance of antenatal care visits among women in Ethiopia. PLoS One. 2017;12(9):e0184934.

4. EEDHS. E. EDHS. Ethiopia demographic and health survey 2016:key indicators. 2016

5. Islam MM, Masud MS. Determinants of frequency and contents of antenatal care visits in Bangladesh: assessing the extent of compliance with the WHO recommendations. PLoS One. 2018;13(9):e0204752.

6. Irena AH, Mwambazi M, Mulenga $\mathrm{V}$. Diarrhea is a major killer of children with severe acute malnutrition admitted to inpatient set-up in Lusaka, Zambia. Nutr J. 2011:10:110.

7. Chamla D, Oladeji O, Maduanusi I, Mele S, Mshelia H, Safi N, et al. High burden of co-morbidity and mortality among severely malnourished children admitted to outpatient therapeutic programme facilities in the conflict setting of Borno, Nigeria: a retrospective review. Time-to-recovery from severe acute malnutrition in children 6-59 months of age enrolled in 
the outpatient treatment program in Shebedino, Southern Ethiopia: a prospective cohort study. Co-morbidity, treatment outcomes and factors affecting the recovery rate of under -five children with severe acute malnutrition admitted in selected hospitals from Ethiopia: retrospective follow up study. Relapse after severe acute malnutrition: A systematic literature review and secondary data analysis. Changes in susceptibility to life-threatening infections after treatment for complicated severe malnutrition in Kenya. Recovery and relapse from severe acute malnutrition after treatment: a prospective, observational cohort trial in Pakistan. Costing of three feeding regimens for home-based management of children with uncomplicated severe acute malnutrition from a randomised trial in India. Effectiveness of a Household Water, Sanitation and Hygiene Package on an Outpatient Program for Severe Acute Malnutrition: A Pragmatic ClusterRandomized Controlled Trial in Chad. An integrated community-based outpatient therapeutic feeding programme for severe acute malnutrition in rural Southern Ethiopia: Recovery, fatality, and nutritional status after discharge. Malnutrition in HIV-Infected Children Is an Indicator of Severe Disease with an Impaired Response to Antiretroviral Therapy. Efficacy of three feeding regimens for home-based management of children with uncomplicated severe acute malnutrition: a randomised trial in India. Treatment outcomes of severe acute malnutrition in children treated within Outpatient. Therapeutic Program (OTP) at Wolaita Zone, Southern Ethiopia: retrospective cross-sectional study. Treatment outcome and factors affecting time to recovery in children with severe acute malnutrition treated at outpatient therapeutic care program. Community-Based Management of Child Malnutrition in Zambia: HIV/AIDS Infection and Other Risk Factors on Child Survival. Seasonal effect and long-term nutritional status following exit from a Community-Based Management of Severe Acute Malnutrition program in Bihar, India. Integrated program achieves good survival but moderate recovery rates among children with severe acute malnutrition in India. Specially formulated foods for treating children with moderate acute malnutrition in low- and middle-income countries. The effectiveness of interventions to treat severe acute malnutrition in young children: a systematic review. A follow-up experience of 6 months after treatment of children with severe acute malnutrition in Dhaka, Bangladesh. Nutritional recovery in HIV-infected and HIV-uninfected children with severe acute malnutrition. Public Health Nutr. 2019;19(1):1-8.

8. Teshome G, Bosha T, Gebremedhin S. Time-to-recovery from severe acute malnutrition in children 6-59 months of age enrolled in the outpatient treatment program in Shebedino, Southern Ethiopia: a prospective cohort study. BMC pediatrics. 2019;19(1):33.

9. Trehan I, HS G, LaGrone LN, Meuli GJ, Wang RJ, Maleta KM, Manary MJ. Antibiotics as part of the management of severe acute malnutrition. $N$ Engl J Med. 2016;28(3).

10. Ngari MM, Thitiri J, Mwalekwa L, Timbwa M, Iversen PO, Fegan GW, et al. The impact of rickets on growth and morbidity during recovery among children with complicated severe acute malnutrition in Kenya: a cohort study. Matern Child Nutr. 2018;14(2):e12569.

11. Derseh B, Mruts K, Demie T, Gebremariam T. Co-morbidity, treatment outcomes and factors affecting the recovery rate of under -five children with severe acute malnutrition admitted in selected hospitals from Ethiopia: retrospective follow up study. Nutr J. 2018;17(1):116.

12. Mengesha MM, Deyessa N, Tegegne BS, Dessie Y. Treatment outcome and factors affecting time to recovery in children with severe acute malnutrition treated at outpatient therapeutic care program. Glob Health Action. 2016;9:30704.

13. Kabalo MY, Seifu CN. Treatment outcomes of severe acute malnutrition in children treated within Outpatient Therapeutic Program (OTP) at Wolaita Zone, Southern Ethiopia: retrospective cross-sectional study. J Health Popul Nutr. 2017;36(1):7.

14. Teshome G, Bosha T, Gebremedhin S. Time-to-recovery from severe acute malnutrition in children 6-59 months of age enrolled in the outpatient treatment program in Shebedino, southern Ethiopia: a prospective cohort study. BMC Pediatr. 2019;19(1):33.

15. Desyibelew HD, Fekadu A, Woldie H. Recovery rate and associated factors of children age 6 to 59 months admitted with severe acute malnutrition at inpatient unit of Bahir Dar Felege Hiwot referral hospital therapeutic feeding unite, Northwest Ethiopia. PLoS One. 2017;12(2):e0171020

16. Doocy S, Tappis H, Villeminot N, Suk A, Kumar D, Fazal S, et al. Point-of-use water treatment improves recovery rates among children with severe acute malnutrition in Pakistan: results from a site-randomized trial. Public Health Nutr. 2018;21(16):3080-90.
17. Yebyo $H G$, Kendall C, Nigusse D, Lemma W. Outpatient therapeutic feeding program outcomes and determinants in treatment of severe acute malnutrition in Tigray, northern Ethiopia: a retrospective cohort study. PLoS One. 2013;8(6):e65840.

18. Massa D, W K , T B, T A. Treatment outcome of severe acute malnutrition and determinants of survival in Northern Ethiopia: A prospective cohort study. Int J Nutr Metab. 2016;8(3):12-23.

19. A M. Predictive factors of time-to-recovery in children with severe acute malnutrition treated in rural area in Mali: an application of Cox regression; 2017.

20. Kokere S, et al. Treatment outcome of SAM treated at pasturalist area, afar, Ethiopia. BMC. 2006.

21. mahama S, Osman SM, Amponsem A, Z J, Abdul-Mumin A, Akanbong $P$, ErnestinaYirkyio, Yakubu E, Ervin S. Treatment outcome of severe acute malnutrition cases at the tamale teaching hospital. J Nutr Metab. 2015;2015:8.

22. Stefania Moramarco GA, Ciarlantini C, Chipoma JK, Simpungwe MK, NielsenSaines K, Palombi L, Buonomo E. Community-based management of child malnutrition in Zambia: HIV/AIDS infection and other risk factors on child survival. Int J Environ Res Public Health. 2016;13(7):666.

23. James P, Sadler K, Wondafrash M, Argaw A, Luo H, Geleta B, Kedir K, Getnet $Y$, Belachew T, Bahwere P. Children with moderate acute malnutrition with no access to supplementary feeding programmes experience high rates of deterioration and no improvement: results from a prospective cohort study in rural Ethiopia. PloS one. 2016;11(4):e0153530.

24. Kabalo MY, Seifu CN. Treatment outcomes of severe acute malnutrition in children treated within Outpatient Therapeutic Program (OTP) at Wolaita Zone, Southern Ethiopia: retrospective cross-sectional study. J Health Popul Nutr. 2017;36(1):7.

25. Y DT, S GY. Survival rate and determinants in treatment of children with severe acute malnutrition using outpatient therapeutic feeding program in Sidama zone, South Ethiopia IOSR. J Mathem (IOSR-JM). 2016;12:3.

26. Gebremichael DY. Predictors of nutritional recovery time and survival status among children with severe acute malnutrition who have been managed in therapeutic feeding centers, Southern Ethiopia: retrospective cohort study. BMC Public Health. 2015;15(1):1267.

27. B REBKHBS. Malnutrition is a determining factor in diarrheal duration, but not incidence, among young children in a longitudinal study in rural Bangladesh. Am J Clin Nutr. 1984;39(1):87-94.

\section{Publisher's Note}

Springer Nature remains neutral with regard to jurisdictional claims in published maps and institutional affiliations.

\section{Ready to submit your research? Choose BMC and benefit from:}

- fast, convenient online submission

- thorough peer review by experienced researchers in your field

- rapid publication on acceptance

- support for research data, including large and complex data types

- gold Open Access which fosters wider collaboration and increased citations

- maximum visibility for your research: over $100 \mathrm{M}$ website views per year

At $\mathrm{BMC}$, research is always in progress.

Learn more biomedcentral.com/submissions 